\title{
AN UNUSUAL CASE OF PULMONARY MUCORMYCOSIS PRESENTING WITH PARAPLEGIA AND SPINAL CORD INFARCTION
}

\author{
Daspin D', Francis Gnanaprakasam², Madan Ramachandran³, Murali Krishnaswami ${ }^{4}$ \\ ${ }_{1}^{1} M B B S, D M R D,(D N B)$, Department of Radiology, MIOT International Hospital, Chennai \\ ${ }^{2} M B B S, D M R D, D N B$, MNAMS, Department of Radiology, MIOT International Hospital, Chennai \\ ${ }_{3} M B B S, M D$, Department of Radiology, MIOT International Hospital, Chennai \\ ${ }^{4} M B B S, M D, P D C C$, Department of Radiology, MIOT International Hospital, Chennai
}

\begin{abstract}
The current study reports a case of a 56-year-old gentleman who presented in our institute with a 3-day history of fever, respiratory distress and acute paraplegia. He is also a known case of uncontrolled type 2 diabetes mellitus, chronic liver disease and chronic obstructive airway disease. Patient was evaluated further and found to have invasive mucormycosis with venous embolism in the paravertebral venous plexus causing vertebral and cord infarction.
\end{abstract}

\section{KEYWORDS}

IPA-Invasive Pulmonary Aspergillosis, COPA-Chronic Pulmonary Aspergillosis, BAL-Bronchoalveolar Lavage, PA -Pulmonary Mucormycosis.

HOW TO CITE THIS ARTICLE: Daspin D, Gnanaprakasam F, Ramachandran M et al. An unusual case of pulmonary mucormycosis presenting with paraplegia and spinal cord infarction. J. Evolution Med. Dent. Sci. 2016;5(76):5665-5667, DOI: $10.14260 /$ jemds/2016/1278

\section{INTRODUCTION}

Pulmonary mucormycosis is an opportunistic pulmonary fungal infection from a fungus belonging to the order Mucorales. It should be distinguished from the related counterpart invasive pulmonary aspergillosis (IPA) as modern first-line antifungals typically used for aspergillosis lack activity against fungi causing mucormycosis. $(1,2)$

It is considered as a rare disease most commonly encountered in patients with prolonged neutropenia. Irrespective of the site of infection, infarction or haemorrhage are the constant features of invasive mycosis.

Various manifestations of pulmonary mucormycosis have been described in the past in literature. $(3,4)$ We report a rare case of pulmonary mucormycosis presenting with acute myelitis and showing spinal cord and vertebral body infarction probably due to air embolism along the venous plexus of the spinal cord.

\section{CASE REPORT}

A 56-year-old gentleman named Mr. Selvaraj with complaints of fever, cough and acute paraplegia for evaluation was admitted in the emergency department in our hospital. He is a known case of type 2 diabetes, decompensated liver disease and chronic obstructive airway disease.

Initial CT imaging of the chest revealed right lower lobe consolidation and loculated effusion with suspicion of infective aetiology; however, the spine imaging of dorsolumbar vertebrae showed air pockets along the vertebral venous plexus within the spinal canal and vertebral bodies from D4-D9 level.(5,2).

Financial or Other, Competing Interest: None.

Submission 12-08-2016, Peer Review 09-09-2016,

Acceptance 15-09-2016, Published 22-09-2016.

Corresponding Author:

Dr. Daspin D,

Department of Radiology,

MIOT Hospital, Manapakkam,

Chennai-600089.

E-mail: daspins@yahoo.com

DOI: $10.14260 /$ jemds $/ 2016 / 1278$
MRI spine of dorsolumbar region showed focal spinal cord infarction at D7 level with vertebral body infarction at D4-D9 level. Patient was shifted to the intensive care unit and followed by critical care team with a team including a pulmonologist and a neurologist. Subsequently, patient deteriorated in a day and has to be intubated. Fibre-optic endoscopy showed copious bronchial red colour secretion in right bronchial tree, mucosa was unhealthy with black pigmentation and BAL was sent for analysis. BAL and tracheal aspirate culture shows heavy growth of mucormycosis and negative for AFB. LP and CSF analysis showed exudate with features suggestive of pyogenic meningitis. Patient was given antibiotics and intravenous infusion of amphotericin. He had worsening metabolic acidosis with lactate 12.8 and worsening renal parameters for which acute peritoneal dialysis was done. Finally, in spite of all ionotropic support and antibiotics, patient died due to septic shock.

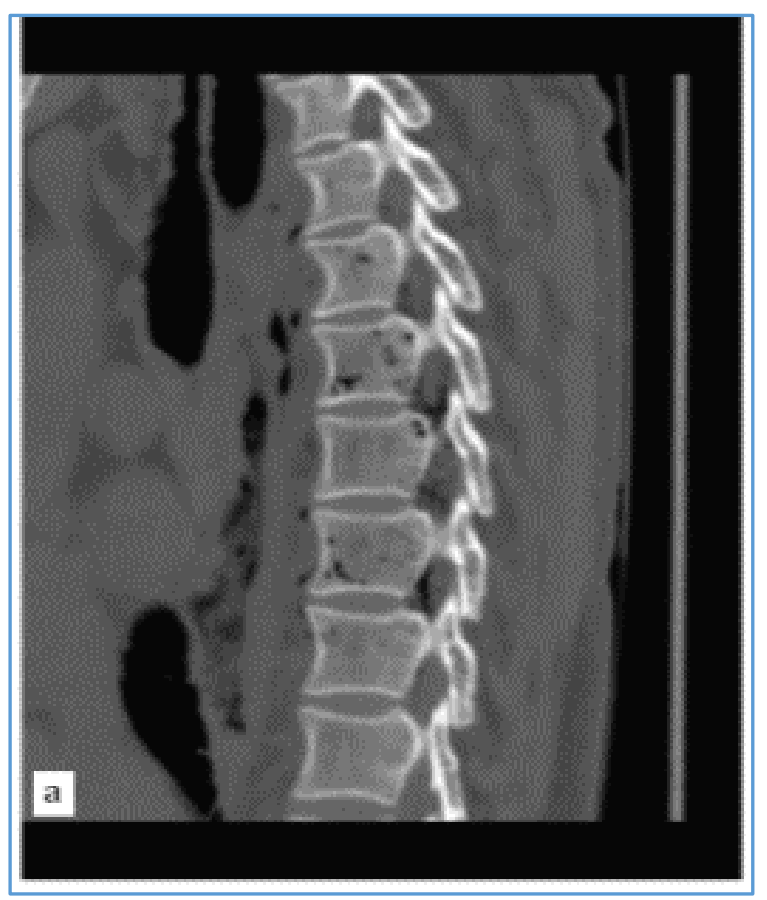



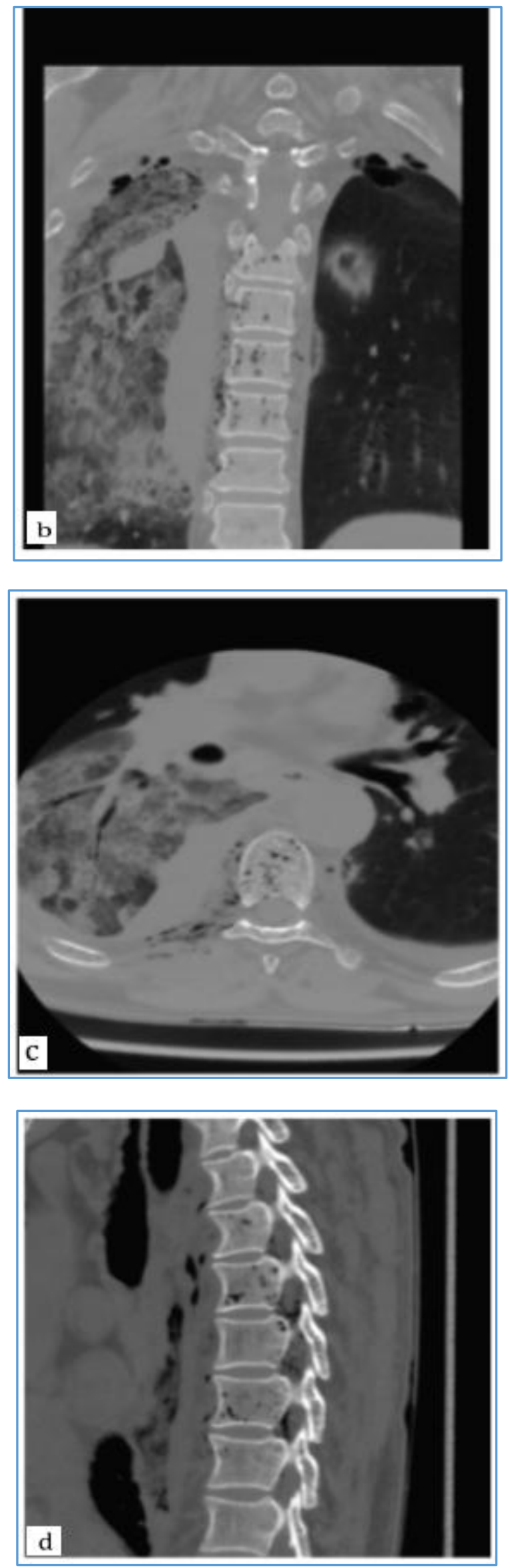

Fig. I (a-d) CT images showing consolidation with ground glass opacities, interstitial thickening in right lung, air pockets along the vertebral venous plexus in spinal canal and vertebral bodies from D4-D8 levels.
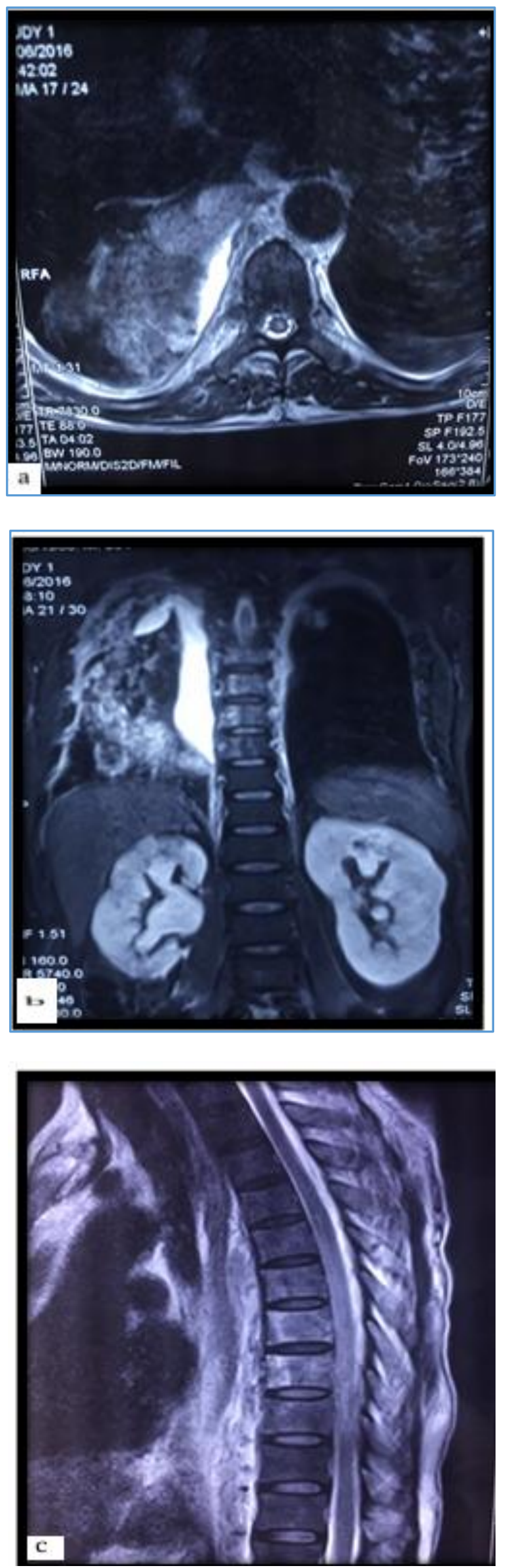


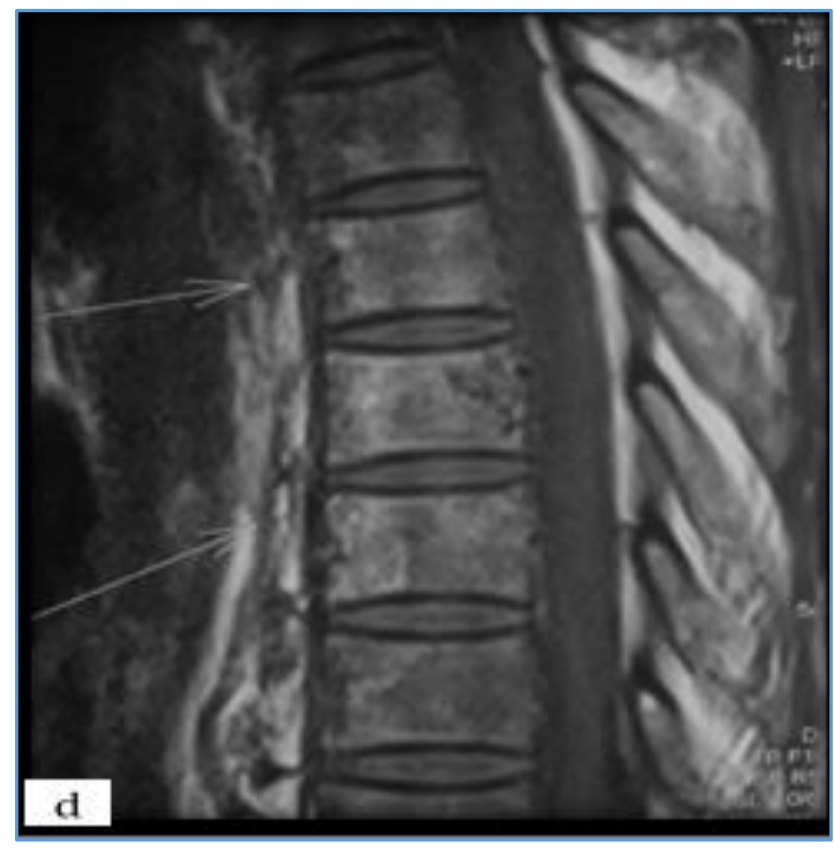

Fig. 2(a-d) MRI images showing abnormal hyperintensity with geographic pattern involving the vertebral body (D4-D8) and focal thoracic cord at $D 7$ level suggestive of infarction

\section{DISCUSSION}

Mucormycosis of the lung is a rare entity seen in immunocompromised patients. Symptoms may comprise fever refractory to broad-spectrum antibiotics, nonproductive cough, and progressive dyspnoea. Pleuritic chest pain, haemoptysis, and pleural effusion are seen less frequently. Invasion of the major pulmonary blood vessels by hyphae may lead to massive, potentially fatal haemoptysis. Invasion of adjacent organs by traversing tissue planes, including the diaphragm, chest wall, and pleura have also been described. It is important to distinguish mucormycosis from aspergillosis because the treatments can differ and because appropriate early treatment of mucormycosis may improve the outcome.(6,5).

The innate immune response to mucormycosis is supplied by mononuclear and polymorphonuclear phagocytes. Only by overcoming these mechanisms can spores germinate into hyphal forms, i.e. the angioinvasive form of infection.(7) Most common predisposing conditions for mucormycosis are uncontrolled diabetes mellitus, malignancy, chronic illnesses and transplants.(8)

Radiographically, a variety of findings may be present. In descending order of frequency, these may include lobar consolidation, isolated masses, nodular disease, and cavitation. High-resolution chest CT scan is the most sensitive method of determining the extent of pulmonary mucormycosis. An important finding is expansion of the mass or consolidation across tissue planes, in particular towards the great vessels in the mediastinum, pleura and paravertebral plexus. $(2,9)$

In this patient, there was significant consolidation with probable invasion of pleura and subsequently paravertebral venous plexus causing disseminated air from the lung causing venous embolism. As a result of this, there is significant cord and vertebral body infarction presenting as paraplegia and sudden respiratory distress. $(2,7)$

\section{Summary}

Pulmonary mucormycosis is a rare infection which when severe can invade tissue planes, pleura and vessels resulting in complications such as acute myelitis with spinal cord and vertebral body infarction. Knowledge of the disease process is needed for emergency management and prevention of complications.

\section{REFERENCES}

1. Van Burik JA, Hare RS, Solomon HF, et al. Posaconazole is effective as salvage therapy in zygomycosis: a retrospective summary of 91 cases. Clin Infect Dis 2006;42(7):e61-e5.

2. Chung JH, Godwin JD, Chien JW, et al. Case 160: pulmonary mucormycosis. Radiology 2010;256(2):667-70.

3. Vogl TJ, Hinrichs T, Jacobi V, et al. Computed tomographic appearance of pulmonary mucormycosis. Rofo 2000;172(7):604-8.

4. Lee FY, Mossad SB, Adal KA. Pulmonary mucormycosis: the last 30 years. Arch Intern Med 1999;159(12):1301-9.

5. Chandler FW, Watts JC. Fungal infections. In: DalI DH, Hammer SP. eds. Pulmonary pathology. New York: Springer-Verlag 1988:228-31.

6. Lehrer RI, Howard DH, Sypherd PS, et al. Mucormycosis. Ann Intern Med 1980;93(1 Pt 1):93-108.

7. Kim N, Barrie J, Raymond G. Residents' corner. Answer to case of the month \#87: pulmonary mucormycosis with angioinvasion of the left subclavian artery. Can Assoc Radiol J 2002;53(5):312-4.

8. Zagoria RJ, Choplin RH, Karstaedt N. Pulmonary gangrene as a complication of mucormycosis. AIR 1985; 144 (6): 1195-6.

9. Libshitz HI, Pagani JJ. Aspergillosis and mucormycosis: two types of opportunistic fungal pneumonia. Radiology 1981;140(2):301-6. 